\title{
Integrated Wireless Multimedia Turbo-Transceiver Design Approaching the Rayleigh Channel's Capacity: Interpreting Shannon's Lessons in the Turbo-Era
}

\author{
S. X. Ng, J. Y. Chung and L. Hanzo \\ School of ECS, University of Southampton, SO17 1BJ, UK. \\ Tel: +44-23-8059 3125, Fax: +44-23-8059 4508 \\ Email: $\{$ sxn,lh\}@ecs.soton.ac.uk, http://www-mobile.ecs.soton.ac.uk
}

\begin{abstract}
Claude Shannon's pioneering work quantified the performance limits of communications systems operating over classic wireline Gaussian channels. However, his source and channel coding theorems were derived for a range of idealistic conditions, which may not hold in low-delay, interactive wireless multimedia communications. Firstly, Shannon's ideal lossless source encoder, namely the entropy encoder may have an excessive codeword length, hence exhibiting a high delay and a high error sensitivity. However, in practice most multimedia source signals are capable of tolerating lossy, rather than lossless delivery to the human eye, ear and other human sensors. The corresponding lossy and preferably low-delay multimedia source codecs however exhibit unequal error sensitivity, which is not the case for Shannon's ideal entropy codec. There are further numerous differences between the Shannonian lessons originally outlined for Gaussian channels and their ramifications for routinely encountered dispersive wireless channels, where typically bursty, rather than random errors are encountered. This paper elaborates on these intriguiging lessons in the context of a few turbo-transceiver design examples, using a jointly optimised turbo transceiver capable of providing unequal error protection in the context of MPEG-4 aided wireless video telephony. The transceiver investigated consists of Space-Time Trellis Coding (STTC) invoked for the sake of mitigating the effects of fading, Trellis Coded Modulation (TCM) or Bit-Interleaved Coded Modulation (BICM) as well as two different-rate Non-Systematic Convolutional codes (NSCs) or Recursive Systematic Convolutional codes (RSCs). A single-class protection based benchmarker scheme combining STTC and NSC is used for comparison with the unequal-protection scheme advocated. The video performance of the various schemes is evaluated when communicating over uncorrelated Rayleigh fading channels. It was found that the achievable performance of the proposed scheme is within $0.99 \mathrm{~dB}$ of the corresponding capacity of the Rayleigh fading channel.
\end{abstract}

\section{SHANNON'S MESSAGE ON SOURCE AND CHANNEL CODING DESIGNED FOR WIRELESS CHANNELS}

The schematic of an integrated multimedia transceiver is seen in Figure 1, which incorporates a source encoder, a channel encoder, an interleaver, and a modulator as well as their inverse functions at the receiver [1]. The ideal source encoder endeavours to remove as much redundancy as possible from the information source signal without affecting its source representation fidelity (i.e. distortionlessly), and it remains oblivious of such practical constraints as a finite delay and limited signal processing complexity. A classic entropy encoder, such as a Huffman encoder [1] constitutes a good example of a lossless source encoder, which is capable of approaching a transmission rate close to the entropy of the source at the cost of an unpredictable encoding delay. Furthermore, since the message becomes undecodable in the presence of transmission errors, regardless of the index of the corrupted bits, all source-encoded bits have an equally high error sensitivity.

By contrast, a practical source encoder used for low-delay interactive multimedia communications will have to retain a limited signal processing complexity and tolerable delay, while attempting to reduce the source representation bit rate to as low a value as possible. This operation seeks to achieve better transmission efficiency, which can be expressed in terms of bit-rate economy or bandwidth conservation. In practise the family of state-of-the-art video source codecs, such as the H.261, H.263 [1], H.264 [1] and MPEG4 [1] codecs are capable of achieving compression ratios in excess 100 by exploiting the psychovisual masking properties of the human eye. Naturally, this is only achievable at the cost of tolerating 'lossy' compression, resulting in perceptually unobjectionable video degradation. Such practical highcompression video source codecs tend to have an unequal sensitivity against transmission errors [1].

The channel encoder re-inserts redundancy or parity information but in a controlled manner in order to allow error correction at the receiver. Since the channel codec is designed for ensuring the best exploitation of the re-inserted redundancy, it is expected to minimise the error probability, when communicating over the most common channel, namely, the so-called Additive White Gaussian Noise (AWGN) channel, which is characterised by a memoryless, random distribution of channel errors. However, for transmission over wireless channels, which have recently become prevalent, the errors tend to occur in bursts owing to the presence of deep received signal fades induced by the destructively superimposed multipath phenomena. This is why our schematic of Figure 1 contains a channel interleaver block, which is included in order to randomise the bursty channel errors. Finally, the modulator is designed to ensure the most bandwidthefficient transmission of the source- and channel-encoded, interleaved information stream, while maintaining the lowest possible bit error probability. The receiver simply carries out the corresponding inverse functions of the transmitter.

In wireless communications over power- and bandlimited channels it is always of prime concern to maintain an optimum compromise in terms of the contradictory requirements of low bit rate, high robustness against channel errors, low delay and low complexity. The minimum bit rate at which distortionless or lossless communications is possible is determined by the entropy of the multimedia source message. Note, however, that in practical terms the source rate corresponding to the entropy is only asymptotically achievable as the encoding memory length or delay tends to infinity. Any further com- 


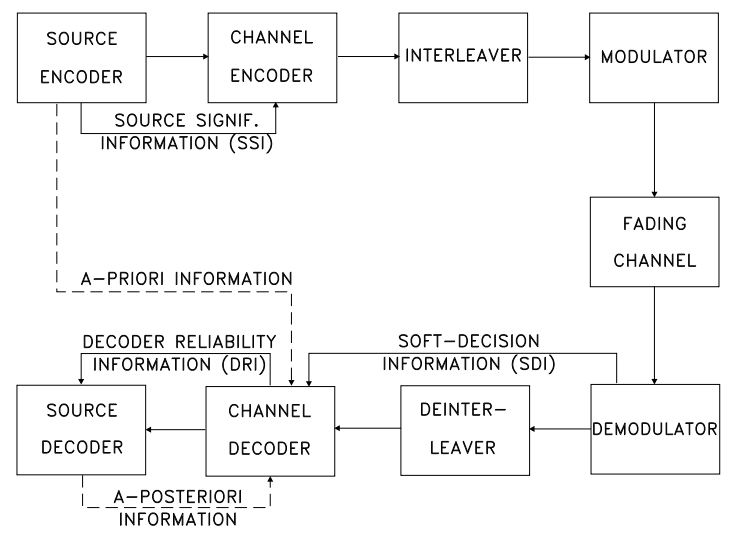

Figure 1: Intelligent transceiver schematic.

pression is associated with information loss or coding distortion. Note that the optimum source encoder generates a perfectly uncorrelated source-coded stream, where all the source redundancy has been removed. Therefore, the entropy-coded symbols are independent and each one has the same significance. Having the same significance implies that the corruption of any of the source-encoded symbols results in identical source signal distortion over imperfect channels.

Under these conditions, according to Shannon's pioneering work [2], which was expanded for example, by Hagenauer [3] and Viterbi [4], the best protection against transmission errors is achieved if source and channel coding are treated as separate entities. When using a block code of length $N$ channel coded symbols in order to encode $K$ source symbols with a coding rate of $R=K / N$, the symbol error rate can be rendered arbitrarily low, if $N$ tends to infinity and the coding rate to zero. This condition also implies encountering an infinite coding delay. Based on the above considerations and on the assumption of additive white Gaussian noise (AWGN) channels, source and channel coding have historically been separately optimised.

By contrast, mobile radio channels are subjected to multipath propagation and hence constitute a more hostile transmission medium than AWGN channels, typically exhibiting path-loss, log-normal slow fading and Rayleigh fast-fading [5]. Furthermore, if the signalling rate used is higher than the channel's coherence bandwidth, over which no spectral-domain linear distortion is experienced, then additional impairments are inflicted by dispersion, which is associated with frequency-domain linear distortions. Under these circumstances the channel's error distribution versus time becomes bursty, and an infinitememory symbol interleaver is required in Figure 1 in order to disperse the bursty channel errors, which renders the error distribution random Gaussian-like, such as the error distribution of AWGN channels. When communicating over bursty Rayleigh-fading mobile channels using low-delay, finite-complexity, systems exploiting either phsycho-visual or phsycho-acoustic masking, many of the above mentioned AWGN-channel related ramifications of Shannon's lessons have limited applicability.

A range of practical limitations must be observed when designing wireless video links. Although it is often possible to further reduce the prevalent typical bit rate of state-of-the-art video codecs, in practical terms this is only possible when using a higher implementational complexity and higher encoding delay. Naturally, the increased al- gorithmic complexity is typically associated with higher power consumption, while the reduced number of bits used for representing a certain video segment intuitively implies that each bit will have an increased relative significance. Accordingly, their corruption may inflict increasingly objectionable video degradations, unless special attention is devoted to this problem [1].

In a somewhat simplistic approach, one may argue that because of the reduced video source rate we may accommodate an increased number of parity symbols using a more powerful, implementationally more complex and lower rate channel codec, while maintaining the same transmission bandwidth. However, the complexity, quality, and robustness trade-off of such a scheme may not always be attractive.

A more intelligent approach is required to design better wireless video transceivers for communicating over mobile radio channels [3]. Again, such an intelligent transceiver is portrayed in Figure 1. Perfect source encoders operating close to the information-theoretical limits of Shannon's predictions can only be designed for stationary video source signals, a condition not satisfied by typical video signals. Further previously mentioned limitations are the encoding complexity and delay. As a consequence of these practical limitations, the video source-coded stream will inherently contain residual redundancy, and the correlated source symbols will exhibit unequal error sensitivity, requiring unequal error protection. Following Hagenauer [3], we will refer to the additional knowledge as regards the different importance or vulnerability of various video-coded bits as source significance information (SSI). Furthermore, Hagenauer termed the confidence associated with the channel decoder's decisions as decoder reliability information (DRI). These additional links between the video source and channel codecs are also indicated in Figure 1. A variety of such techniques have successfully been used in robust voice and video sourcesensitivity matched channel coding $[1,6]$.

The paper is structured as follows. In Section 3 we describe the proposed system's architecture and highlight the interactions of its constituent elements. We elaborate further by characterising the achievable system performance in Section 4 and conclude with a range of system design guideline in Section 5 .

\section{MOTIVATION AND BACKGROUND}

In this study the state-of-the-art MPEG-4 video codec was incorporated in a sophisticated unequal-protection turbo transceiver using joint coding and modulation as inner coding [7], twin-class convolutional outer coding [7] as well as space time coding based spatial diversity [7]. Specifically, Trellis Coded Modulation (TCM) [7, 8], which employs a Recursive Systematic Convolutional code (RSC), constitutes a bandwidth-efficient joint channel coding and modulation scheme, which was originally designed for transmission over Additive White Gaussian Noise (AWGN) channels. By contrast, BitInterleaved Coded Modulation (BICM) [9] employing parallel bitbased interleavers was designed for communicating over uncorrelated Rayleigh fading channels. Therefore, TCM outperforms BICM when communicating over AWGN channels since TCM exhibits a higher Euclidean distance. By contrast, the opposite is true, when communicating over uncorrelated Rayleigh fading channels, since BICM exhibits a higher Hamming distance. Space-Time Trellis Coding (STTC) [10] employing multiple transmit and receive antennas was also invoked for providing spatial diversity gain. Furthermore, maximal minimum distance Non-Systematic Convolutional codes (NSCs) [11, p. 331] having two different code-rates were used as outer encoders for providing unequal video protection. We also study the performance of the proposed system that employs two different-rate RSCs as the outer encoders. 


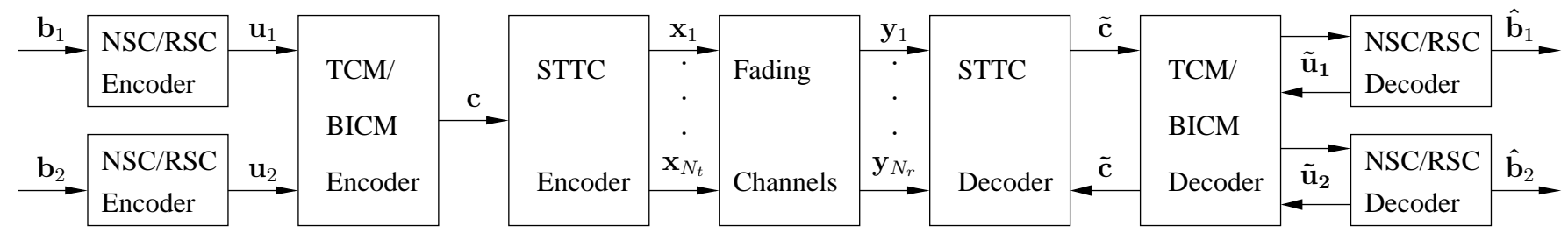

Figure 2: Block diagram of the serially concatenated STTC-TCM/BICM-2NSC/2RSC scheme. The notations $\mathbf{b}_{i}, \mathbf{u}_{i}, \mathbf{c}, \mathbf{x}_{j}$ and $\mathbf{y}_{k}$ denote the vectors of the class- $i$ video bits, the encoded bits of class- $i$ NSC/RSC encoder, the TCM encoded symbols (or BICM encoded bits), the STTC encoded symbols for transmitter $j$ and the received symbols at receiver $k$, respectively. Furthermore, the notations $(\hat{.})$ and $(\tilde{(.)}$ denote the estimate of (.) and the extrinsic information regarding (.), respectively. The number of transmitters and receivers is denoted as $N_{t}$ and $N_{r}$, respectively. The symbol-based (or bit-based) channel interleaver between the STTC and TCM (or BICM) schemes as well as the two bit-based interleavers between the TCM and NSC/RSC schemes are not shown for simplicity.

Again, in this contribution, a novel unequal-protection joint MPEG4 source-coding, channel-coding, modulation and spatial diversity aided turbo-transceiver is investigated, which consists of a STTC, a TCM and two different-rate NSCs/RSCs, which will be referred to as the STTC-TCM-2NSC or STTC-TCM-2RSC scheme. Note that when the spacial diversity order is sufficiently high, the channel's Rayleigh fading envelope is transformed to a Gaussian-like near-constant envelope. Hence, the benefits of a TCM scheme designed for AWGN channels will be efficiently exploited, when TCM is concatenated with STTC in comparison to BICM [12]. We will also investigate the STTC-BICM-2RSC scheme, where BICM is employed as the inner code for the sake of studying the performance difference between BICM and TCM as the inner code in the STTC-based unequalprotection turbo transceiver. We will also demonstrate that significant iteration gains are attained with the aid of the proposed turbo transceiver.

\section{THE TURBO TRANSCEIVER}

The schematic of the serially concatenated STTC-TCM/BICM-2NSC/ 2RSC turbo scheme using a STTC, a TCM/BICM and two NSCs/RSCs as its constituent codes is depicted in Figure 2. The MPEG-4 codec operated at $R_{f}=30$ frames per second using the $(176 \times 144)$-pixel Quarter Common Intermediate Format Miss America video sequence, encoded at a near-constant bitrate of $R_{b}=69 \mathrm{kbps}$. Hence, we have $R_{b} / R_{f}=2300$ bits per video frame. We partition the video bits into two unequal protection classes. Specifically, class- 1 and class-2 consist of $25 \%$ (which is 575 bits) and $75 \%$ (which is 1725 bits) of the total number of video bits. The more sensitive video bits constituted mainly by the MPEG-4 framing and synchronisation bits are in class- 1 and they are protected by a stronger binary NSC/RSC having a coding rate of $R_{1}=k_{1} / n_{1}=1 / 2$ and a code memory of $L_{1}=3$. The less sensitive video bits predominantly signalling the MPEG-4 Discrete Cosine Transform (DCT) coefficients and motion vectors are in class- 2 and they are protected by a weaker non-binary NSC/RSC having a coding rate of $R_{2}=k_{2} / n_{2}=3 / 4$ and a code memory of $L_{2}=3$. Note that the number of MPEG-4 framing and synchronisation bits is only about $10 \%$ of the total video bits. Hence, about $25 \%-10 \%=15 \%$ of class- 1 bits are constituted by the video bits signalling the most sensitive MPEG-4 DCT coefficients. We invoke code termination bits in both NSCs/RSCs and hence the number of coded bits emerging from the $R_{1}=1 / 2$ binary NSC/RSC encoder is $\left(575+k_{1} L_{1}\right) / R_{1}=1156$ bits, while that generated by the $R_{2}=3 / 4$ non-binary NSC/RSC encoder is $\left(1725+k_{2} L_{2}\right) / R_{1}=2312$ bits.

The class- 1 and class- 2 NSC/RSC coded bit sequences are interleaved by two separate bit interleavers of length 1156 and 2312 bits, respectively. The two interleaved bit sequences are then concatenated to form a bit sequence of $1156+2312=3468$ bits. This bit sequence is then fed to the TCM/BICM encoder having a coding rate of $R_{3}=k_{3} / n_{3}=3 / 4$ and a code memory of $L_{4}=3$. Note that TCM employs a Set Partitioning (SP) based bit mapper [8], hence the Most Significant Bit (MSB) of the three-bit input symbol of the rate-3/4 TCM encoder has a higher protection. Therefore, we map the interleaved bit sequence of the class-1 NSC/RSC encoder to the MSB of the TCM scheme's three-bit input symbol for the sake of further protecting the class- 1 video bits. We employ code termination also in the TCM/BICM scheme and hence at the TCM/BICM encoder's output we have $\left(3468+k_{3} L_{3}\right) / R_{3}=4636$ bits or $4636 / 4=1159$ symbols. The TCM symbol sequence (or BICM bit sequence) is then symbol-interleaved (or bit-interleaved) and fed to the STTC encoder. We invoke a 16-state STTC scheme having a code memory of $L_{4}=4$ and $N_{t}=2$ transmit antennas, employing $M=16$-level Quadrature Amplitude Modulation (16QAM). We terminate the STTC code by a 4-bit 16QAM symbol, since we have $N_{t}=2$. Therefore, at each transmit antenna we have $1159+1=1160$ 16QAM symbols or $4 \times 1160=4640$ bits in a transmission frame. The overall coding rate is given by $R=2300 / 4640=0.496$ and the effective throughput of the system is $\log _{2}(M) R=1.98$ Bits Per Symbol (BPS).

The STTC decoder employed $N_{r}=2$ receive antennas and the received signals are fed to the iterative decoders for the sake of estimating the video bit sequences in both class- 1 and class-2, as seen in Figure 2. More explicitly, the extrinsic information regarding the TCM encoded symbol sequence (or BICM encoded bit sequence) $\mathbf{c}$ is passed between the STTC and TCM/BICM decoders. By contrast, the extrinsic information regarding the two NSC/RSC encoded bit sequences, i.e. $\mathbf{u}_{1}$ and $\mathbf{u}_{2}$, is passed between the TCM/BICM and NSC/RSC decoders. Let us define a single decoding iteration as a combination of a STTC decoding, a TCM/BICM decoding, a class$1 \mathrm{NSC} / \mathrm{RSC}$ decoding and a class-2 NSC/RSC decoding step for the STTC-TCM/BICM-2NSC/2RSC scheme.

For the sake of benchmarking the scheme advocated, we created a powerful benchmark scheme by replacing the TCM/BICM and NSC/RSC encoders of Figure 2 by a single NSC codec having a coding rate of $R_{0}=k_{0} / n_{0}=1 / 2$ and a code memory of $L_{0}=6$. We will refer to this benchmarker scheme as the STTC-NSC arrangement. All video bits are equally protected in the benchmarker scheme by a single NSC encoder and a STTC encoder. A bit-based channel interleaver is inserted between the NSC encoder and STTC encoder. Taking into account the bits required for code termination, the number of output bits of the NSC encoder is $\left(2300+k_{0} L_{0}\right) / R_{0}=4612$, which corresponds to 1153 16QAM symbols. Again, a 16-state STTC scheme having $N_{t}=2$ transmit antennas is employed. After code termination, we have $1153+1=1154$ 16QAM symbols or $4(1154)=$ 4616 bits in a transmission frame at each transmit antenna. Similar to 
the STTC-TCM/BICM-2NSC/2RSC scheme, the overall coding rate is given by $R=2300 / 4616=0.498$ and the effective throughput is $\log _{2}(16) R=1.99$ BPS, both of which are close to the corresponding values of the proposed scheme. A decoding iteration of the STTCNSC benchmarker scheme is comprised of a STTC decoding and a NSC decoding step.

We will quantify the decoding complexity of the proposed STTCTCM/BICM-2NSC/2RSC scheme and that of the benchmarker scheme using the number of decoding trellis states. The total number of decoding trellis states per iteration of the proposed scheme employing $2 \mathrm{NSC} / \mathrm{RSC}$ decoders having a code memory of $L_{1}=L_{2}=3$, TCM/BICM having $L_{3}=3$ and STTC having $L_{4}=4$, is $S=$ $2^{L_{1}}+2^{L_{2}}+2^{L_{3}}+2^{L_{4}}=40$. By contrast, the total number of decoding trellis states per iteration for the benchmarker scheme having a code memory of $L_{0}=6$ and STTC having $L_{4}=4$, is given by $S=2^{L_{0}}+2^{L_{4}}=80$.

\section{SIMULATION RESULTS}

We continue our discourse by characterising the attainable performance of the proposed MPEG-4 based video telephone schemes using both the Bit Error Ratio (BER) and the average video Peak Signal to Noise Ratio (PSNR) [1].

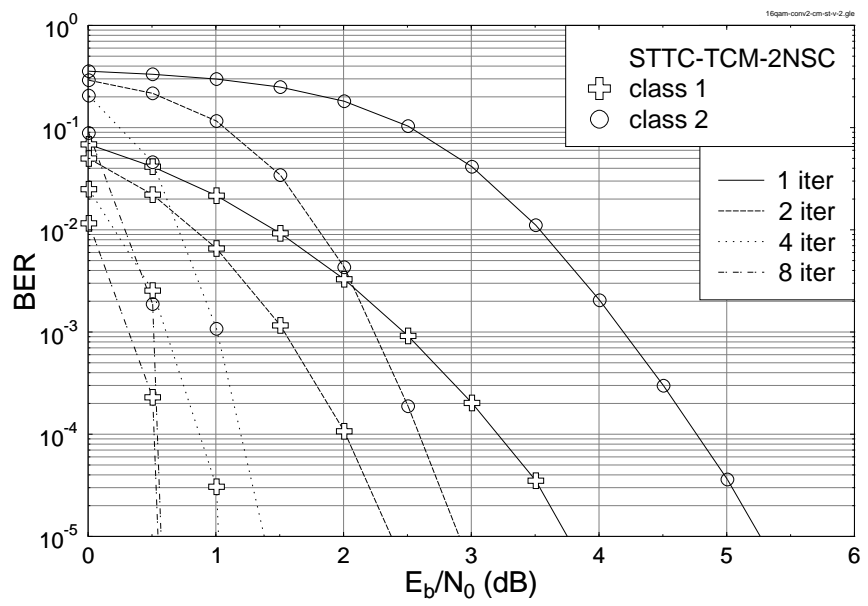

Figure 3: BER versus $E_{b} / N_{0}$ performance of the 16QAM-based STTC-TCM-2NSC assisted MPEG-4 scheme, when communicating over uncorrelated Rayleigh fading channels. The effective throughput was $1.98 \mathrm{BPS}$.

Figures 3 and 4 depict the class- 1 and class-2 BER versus Signal to Noise Ratio (SNR) per bit, namely $E_{b} / N_{0}$, performance of the 16QAM-based STTC-TCM-2NSC and STTC-TCM-2RSC schemes, respectively, when communicating over uncorrelated Rayleigh fading channels. Specifically, the class-1 bits benefit from more than an order of magnitude lower BER at a given SNR, than the class-2 bits. Figure 5 compares the overall BER performance of the STTC-TCM2NSC and STTC-TCM-2RSC schemes. More explicitly, the STTCTCM-2RSC scheme is outperformed by the STTC-TCM-2NSC arrangement, when the number of iterations is lower than eight. An approximately $4 \mathrm{~dB}$ and $6 \mathrm{~dB}$ iteration gain was attained by the STTCTCM-2NSC and STTC-TCM-2RSC schemes, respectively, when the number of iterations was increased from one to eight at $\mathrm{BER}=10^{-4}$. Note in Figures 4 and 5 that the STTC-TCM-2RSC scheme suffers from an error floor, despite having a high iteration gain, which is due to the employment of RSC outer codes instead of the NSC outer

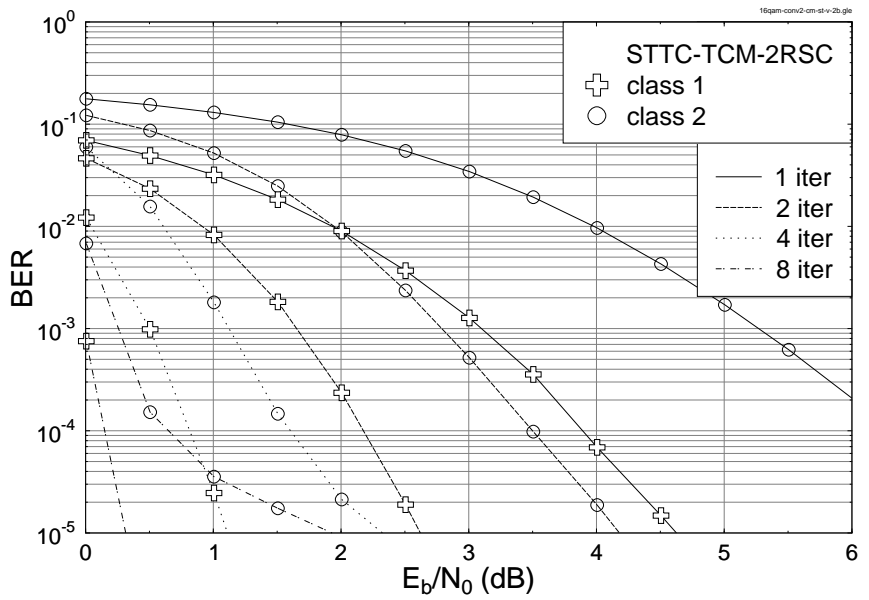

Figure 4: BER versus $E_{b} / N_{0}$ performance of the 16QAM-based STTC-TCM-2RSC assisted MPEG-4 scheme, when communicating over uncorrelated Rayleigh fading channels. The effective throughput was $1.98 \mathrm{BPS}$.

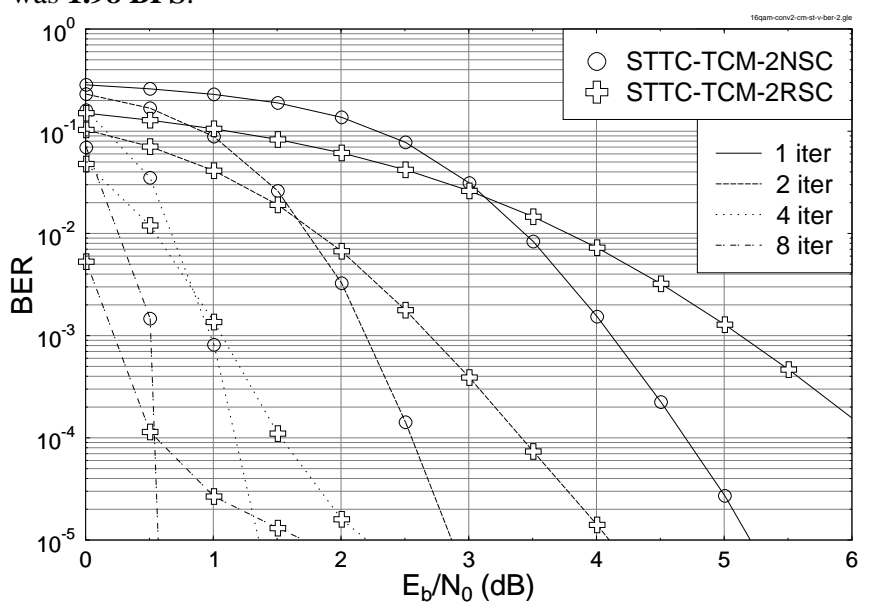

Figure 5: BER versus $E_{b} / N_{0}$ performance of the 16QAMbased STTC-TCM-2NSC and STTC-TCM-2RSC assisted MPEG-4 schemes, when communicating over uncorrelated Rayleigh fading channels. The effective throughput was 1.98 BPS.

codes.

The BER performance curves of STTC-BICM-2RSC and STTCNSC are shown in Figure 6. Note that if we reduce the code memory of the NSC constituent code of the STTC-NSC benchmarker scheme from $L_{0}=6$ to 3 , the best possible performance becomes poorer. If we increased $L_{0}$ from 6 to 7 (or higher), the decoding complexity increased significantly, while the attainable best possible performance is only marginally increased. Hence, the STTC-NSC scheme having $L_{0}=6$ constitutes a powerful benchmarker scheme in terms of its performance versus complexity tradeoffs. As observed in Figure 6, the performance of the STTC-BICM-2RSC scheme is even worse than that of the STTC-NSC benchmarker scheme. More explicitly, STTC-BICM-2RSC employing eight iterations cannot outperform the STTC-NSC arrangement employing two iterations. By changing the outer code to NSC, i.e. using the STTC-BICM-2NSC scheme, the attainable performance cannot be further improved. The complexity of the STTC-TCM-2NSC/2RSC arrangement having four (or eight) 


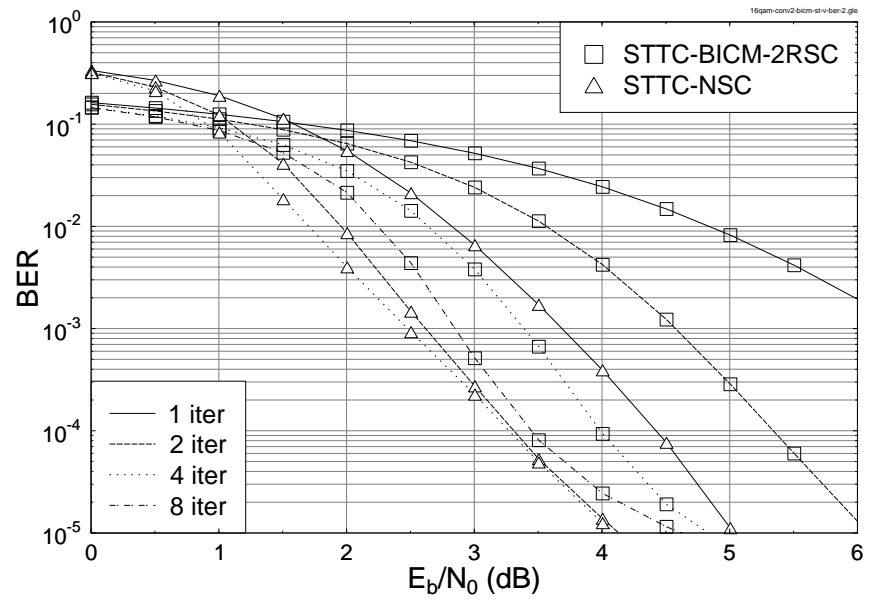

Figure 6: BER versus $E_{b} / N_{0}$ performance of the 16QAM-based STTC-BICM-2RSC and STTC-NSC assisted MPEG-4 schemes, when communicating over uncorrelated Rayleigh fading channels. The effective throughput of STTC-BICM-2RSC and STTC-NSC was 1.98 BPS and 1.99 BPS, respectively.

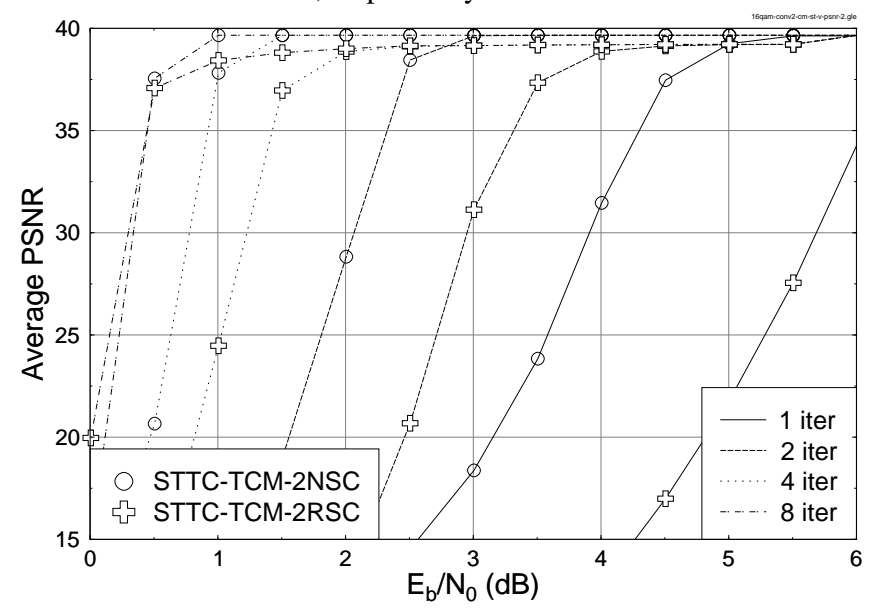

Figure 7: Average PSNR versus $E_{b} / N_{0}$ performance of the proposed 16QAM-based STTC-TCM-2NSC and STTC-TCM-2RSC assisted MPEG-4 schemes, when communicating over uncorrelated Rayleigh fading channels. The effective throughput was 1.98 BPS.

iterations corresponds to 160 (or 320) trellis states, which is similar to that of the STTC-NSC scheme having two (or four) iterations. Hence at a complexity of 160 (or 320) trellis states, the BER performance of the STTC-TCM-2NSC (or STTC-TCM-2RSC) scheme is approximately $2 \mathrm{~dB}$ (or $2.8 \mathrm{~dB}$ ) better than that of the STTC-NSC benchmarker scheme at $\mathrm{BER}=10^{-4}$.

Let us now consider the PSNR versus $E_{b} / N_{0}$ performance of the systems characterised in Figures 7 and 8 . The PSNR performance trends are similar to our observations made in the context of the achievable BER results. The maximum attainable PSNR is $39.7 \mathrm{~dB}$. Observe in Figure 7 that the BER error floor of the STTC-TCM-2RSC scheme resulted in a slightly lower maximum attainable PSNR value, when we had $E_{b} / N_{0}<6 \mathrm{~dB}$. Furthermore, when employing eight iterations at $E_{b} / N_{0}=0.5 \mathrm{~dB}$, the PSNR of STTC-TCM-2RSC was found to be slightly lower than that of the STTC-TCM-2NSC arrangement, although the BER of STTC-TCM-2RSC is significantly lower

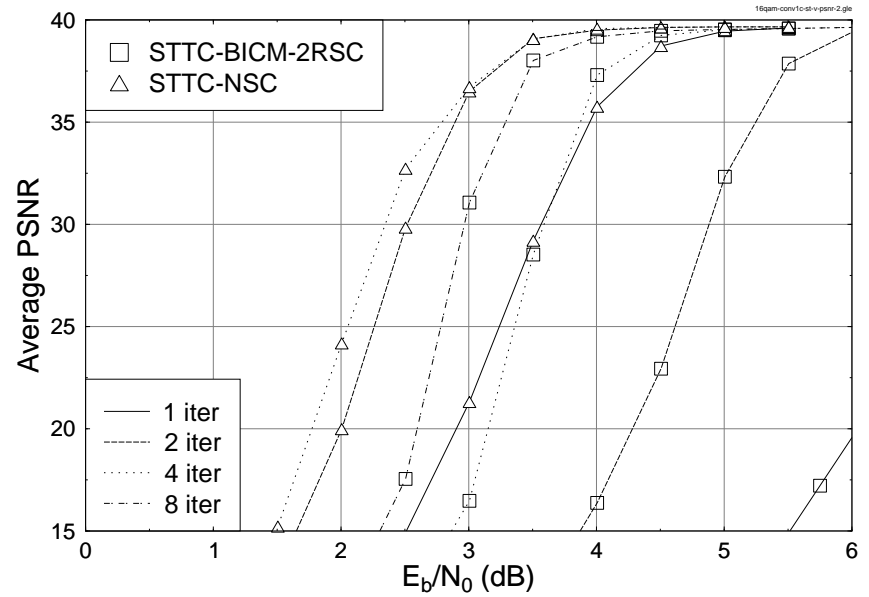

Figure 8: Average PSNR versus $E_{b} / N_{0}$ performance of the 16QAMbased STTC-BICM-2RSC and STTC-NSC assisted MPEG-4 benchmarker schemes, when communicating over uncorrelated Rayleigh fading channels. The effective throughput of STTC-BICM-2RSC and STTC-NSC was 1.98 BPS and 1.99 BPS, respectively.

than that of the STTC-TCM-2NSC scheme, as it is evidenced in Figure 5. This is because STTC-TCM-2RSC suffers from a higher frame error ratio, despite having a lower BER, in comparison to the STTCTCM-2NSC scheme at $E_{b} / N_{0}=0.5 \mathrm{~dB}$.

Let us finally compare the attainable performance of the STTCTCM-2NSC/2RSC and STTC-NSC schemes to the Rayleigh channel's capacity. More explicitly, the Multi-Input Multi-Output (MIMO) channel capacity for a spacetime coding system achieving diversity gain but no multiplexing gain is expressed as [13]:

$$
C=\log _{2}(M)-\frac{1}{M} \sum_{m=1}^{M} E\left[\log _{2} \sum_{i=1}^{M} \exp \left(\Phi_{i}^{m}(N)\right)\right]
$$

where $M$ is the number of modulation levels, $N=N_{t} \times N_{r}$ and $\Phi_{i}^{m}(N)$ is given by [13]:

$$
\Phi_{i}^{m}(N)=\frac{-\left|\chi_{2 N}^{2}\left(\mathbf{x}_{m}-\mathbf{x}_{i}\right)+\boldsymbol{\Omega}\right|^{2}+|\boldsymbol{\Omega}|^{2}}{\chi_{2 N}^{2} N_{0}}
$$

where $\mathbf{x}_{m}$ is the $m$ th phasor in the $M$-ary modulation constellation, $N_{0} / 2$ is the noise variance per dimension, $\chi_{2 N}^{2}=\sum_{n=1}^{N}\left|\mathbf{h}_{n}\right|^{2}$ represents the chi-squared distributed random variable having $2 N$ degree of freedom, $\mathbf{h}_{n}$ is the $n$th link of the complex-valued MIMO channels and $\Omega$ is the resultant MIMO channel's complex-valued noise having a zero mean and a variance of $\chi_{2 N}^{2} N_{0} / 2$ per dimension. Figure 9 shows the the MIMO channel capacity limit for a spacetime coding system employing 16QAM and $N_{t}=N_{r}=2$. More specifically, the MIMO channel capacity limit at a throughput of 1.98 BPS and $1.99 \mathrm{BPS}$ is $E_{b} / N_{0}=-0.49 \mathrm{~dB}$ and $-0.47 \mathrm{~dB}$, respectively. Hence, the achievable performance of the STTC-TCM-2RSC scheme is only $0.99 \mathrm{~dB}$ away from the Rayleigh channel's capacity limit of $-0.49 \mathrm{~dB}$ (1.98 BPS) at PSNR $>37 \mathrm{~dB}$ and $\mathrm{BER}=10^{-4}$ after the eighth turbo iteration. By contrast, the STTC-NSC benchmarker scheme's performance curve is $3.67 \mathrm{~dB}$ away from the corresponding channel capacity limit of $-0.47 \mathrm{~dB}(1.99 \mathrm{BPS})$ at PSNR $>37 \mathrm{~dB}$ and $\mathrm{BER}=10^{-4}$ after the second/fourth iteration. 


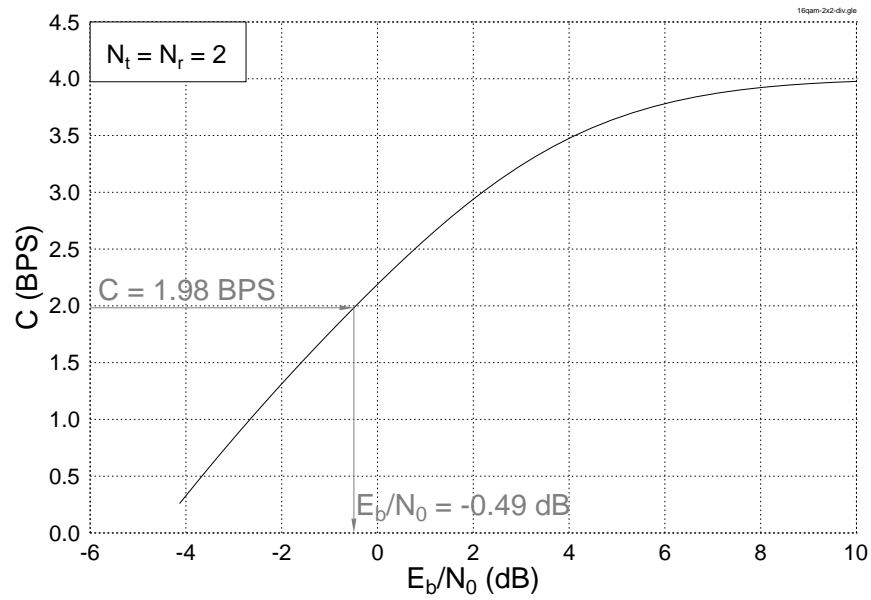

Figure 9: The MIMO channel capacity limit for a spacetime coding system, which achieves diversity gain but no multiplexing gain, employing 16QAM and $N_{t}=N_{r}=2$.

\section{CONCLUSIONS}

In conclusion, a jointly optimised source-coding, outer channel-coding, inner coded modulation and spatial diversity aided turbo transceiver was proposed for MPEG-4 wireless video telephony. With the aid of two different-rate NSCs/RSCs the video bits were protected differently according to their sensitivity. The employment of TCM improved the bandwidth efficiency of the system and by utilising STTC spatial diversity was attained. The performance of the proposed STTCTCM-2NSC/STTC-TCM-2RSC scheme was enhanced with the advent of an efficient iterative decoding structure. It was shown in Figures 5 and 7 that the STTC-TCM-2RSC scheme required $E_{b} / N_{0}=$ $0.5 \mathrm{~dB}$ in order to attain $\mathrm{BER}=10^{-4}$ and PSNR $>37 \mathrm{~dB}$, which is only $0.99 \mathrm{~dB}$ away from the corresponding channel capacity. Furthermore, at the cost of a similar complexity, the STTC-TCM-2RSC scheme is approximately $2.8 \mathrm{~dB}$ more efficient in terms of the required $E_{b} / N_{0}$ than the STTC-NSC benchmarker scheme. Our future research aims for designing similarly powerful schemes approaching the Rayleigh fading channel's capacity for audio and voice systems.

\section{ACKNOWLEDGEMENTS}

The financial support of both the EPSRC, Swindon UK and the EU under the auspices of the Phoenix project is gratefully acknowledged.

\section{REFERENCES}

[1] L. Hanzo, P. Cherriman, and J. Streit, Wireless Video Communications: From Second to Third Generation Systems, WLANs and Beyond. IEEE Press and John Wiley, 2001 (For detailed contents please refer to http://www-mobile.ecs.soton.ac.uk.), 2001.

[2] C. Shannon, Mathematical Theory of Communication. University of Illinois Press, 1963.

[3] J. Hagenauer, "Quellengesteuerte kanalcodierung fuer sprach- und tonuebertragung im mobilfunk," Aachener Kolloquium Signaltheorie, pp. 67-76, 23-25 March 1994.

[4] A. Viterbi, "Wireless digital communications: A view based on three lessons learned," IEEE Communications Magazine, pp. 33-36, September 1991.

[5] D. Greenwood and L. Hanzo, "Characterisation of mobile radio channels," in Mobile Radio Communications (R. Steele and L. Hanzo, eds.), ch. 2, pp. 92-185, Piscataway, NJ: IEEE Press, 1999.
[6] L. Hanzo, F. Somerville, and J. Woodard, Voice Compression and Communications: Principles and Applications for Fixed and Wireless Channels. IEEE Press and John Wiley, 2001 (For detailed contents, please refer to http://www-mobile.ecs.soton.ac.uk.).

[7] L. Hanzo, T.H. Liew and B.L. Yeap, Turbo Coding, Turbo Equalisation and Space Time Coding for Transmission over Wireless channels. New York, USA: John Wiley IEEE Press, 2002.

[8] G. Ungerboeck, "Channel coding with multilevel/phase signals," IEEE Transactions on Information Theory, vol. IT-28, pp. 55-67, January 1982.

[9] E. Zehavi, "8-PSK trellis codes for a Rayleigh fading channel," IEEE Transactions on Communications, vol. 40, pp. 873-883, May 1992.

[10] V. Tarokh, N. Seshadri and A. R. Calderbank, "Space-time Codes for High Rate Wireless Communication: Performance analysis and code construction," IEEE Transactions on Information Theory, vol. 44, pp. 744-765, March 1998.

[11] S. Lin and D. Constello Jr., Error Control Coding: Fundamentals and Applications. Englewood Cliffs, NJ: Prentice-Hall, October 1982.

[12] S. X. Ng, F. Guo and L. Hanzo, "Iterative Detection of Diagonal Block Space Time Trellis Codes, TCM and Reversible Variable Length Codes for Transmission over Rayleigh Fading Channels," in IEEE Vehicular Technology Conference, (Los Angeles, USA), 26-29 September 2004.

[13] S. X. Ng and L. Hanzo, "On the MIMO Channel Capacity of MultiDimensional Signal Sets," in IEEE Vehicular Technology Conference, (Los Angeles, USA), 26-29 September 2004. 